\title{
A Self-Learning Methodology for Epileptic Seizure Detection with Minimally-Supervised Edge Labeling
}

\author{
Damián Pascual, Amir Aminifar, David Atienza \\ Embedded Systems Lab. (ESL), EPFL, Switzerland \\ \{damian.pascual, amir.aminifar, david.atienza\} @epfl.ch
}

\begin{abstract}
Epilepsy is one of the most common neurological disorders and affects over 65 million people worldwide. Despite the continuing advances in anti-epileptic treatments, one third of the epilepsy patients live with drug resistant seizures. Besides, the mortality rate among epileptic patients is $2-3$ times higher than in the matching group of the general population. Wearable devices offer a promising solution for the detection of seizures in real time so as to alert family and caregivers to provide immediate assistance to the patient. However, in order for the detection system to be reliable, a considerable amount of labeled data is needed to train it. Labeling epilepsy data is a costly and time-consuming process that requires manual inspection and annotation of electroencephalogram (EEG) recordings by medical experts. In this paper, we present a self-learning methodology for epileptic seizure detection without medical supervision. We propose a minimally-supervised algorithm for automatic labeling of seizures in order to generate personalized training data. We demonstrate that the median deviation of the labels from the ground truth is only 10.1 seconds or, equivalently, less than $1 \%$ of the signal length. Moreover, we show that training a real-time detection algorithm with data labeled by our algorithm produces a degradation of less than $2.5 \%$ in comparison to training it with data labeled by medical experts. We evaluated our methodology on a wearable platform and achieved a lifetime of 2.59 days on a single battery charge.
\end{abstract}

\section{INTRODUCTION}

Epilepsy is a chronic neurological disorder that affects over 65 million people around the world [1]. Despite being the fourth most common chronic disorder [2], the understanding of its causes is still limited. Although substantial advances have been made in the field of anti-epileptic drugs, one third of the epilepsy patients live with uncontrolled seizures [1].

Epilepsy is manifested by recurrent unprovoked seizures due to abnormal neuronal activity in the brain [1]. The length of the seizures can range from few seconds to several minutes and the symptoms include behavioral arrest, rigid extension of limbs, automatic movements and severe body convulsions [3]. The unpredictability of these symptoms not only degrades the quality of life of the patient, but could also be lifethreatening, e.g. driving accidents or drowning. Besides, in the most severe cases, an epileptic seizure may result in sudden unexpected death in epilepsy (SUDEP). As a consequence, mortality among people with epilepsy is $2-3$ times higher than in the age- and sex-matched general population [4].

Due to the unpredictability of the seizures, today, the most promising way of reducing mortality and improving the life standard of the patients is continuous real-time monitoring.

This work has been partially supported by the Human Brain Project (HBP) SGA2 (GA No. 785907), the MyPreHealth research project (Hasler Foundation project No. 16073), and the WiTNESS project (Promobilia Foundation project No. 18079).
Continuous monitoring enables alerting the family members and caregivers in order to assist the person whenever a seizure occurs. However, traditional patient monitoring takes place in the hospitals over several days and in an intrusive manner involving very bluky medical devices [5].

Wearable technologies open up the possibility of bringing the monitoring outside the hospital environment to the patients' daily life by removing the dependence on the bulky medical equipment. Ultra-low power portable devices (also known as edge devices) can function over extended periods of time capturing and processing biological signals in order to detect epileptic seizures early and raise alerts.

The state-of-the-art algorithms for epileptic seizure detection are based on machine learning techniques [6] and hence, they require a considerable amount of labeled data for the training. However, labeling recordings of epileptic seizures is a very costly and time-consuming process that requires manual inspection and annotation of EEG signals by medical experts. On top of this, the variability of the brain signals across patients significantly degrades the classification performance between generic and personalized approaches [7].

To address these two issues, we propose a new methodology that labels epileptic seizures in edge devices by detecting them a posteriori with only minimal supervision from the patient. The personalized data generated is used to train a real-time detection algorithm. If a seizure is missed, the patient enters into a state of impaired consciousness without any call for assistance being sent. Most people recover from this state within an hour [8], [9]. Upon recovery, the patient touches a button on a smartwatch/smartphone indicating that a seizure occurred in the last hour. Then, our proposed algorithm detects and labels the seizure, and this labeled data is used to train the real-time classifier. In this manner, with every missed seizure our methodology generates personalized data and the real-time detection algorithm becomes more robust.

In this work, we address the problem of collecting and labeling personalized data at the edge device and without medical supervision for training a real-time epileptic seizure detection algorithm. The main contributions are:

- Conception of a self-learning methodology for epileptic seizure detection and demonstration of a minimally supervised algorithm for labeling epileptic seizures at the edge device. The median deviation of the labels given by this algorithm is only 10.1 seconds from the ground truth.

- Validation of the proposed self-learning methodology, in which the real-time detection degrades by only $2.5 \%$ in comparison to using expert-labeled data, and evaluation 
of the battery lifetime of a wearable device running our methodology, which achieves 2.59 days of operation on one charge.

\section{RELATED WORK}

The most common wearable system to capture brain activity is the EEG cap with embedded electrodes [10] placed according to the international 10-20 system [11]. Based on recordings obtained using the EEG cap, many different seizure detection methods have been proposed in the literature [6].

Supervised learning techniques exist that use a number of different approaches such as discrete wavelets [12], Fourier transform [13], support vector machines (SVM) [14], and neural networks [15], [16]. In spite of achieving high classification performance, these methodologies require large amounts of training data which is generally not available for new patients. The medical experts then need to inspect this large amount of data carefully in a lengthy process by manually going through the video-EEG (vEEG) or EEG to annotate the onset/offset of the seizures for each patient, which represents a limiting factor associated with the supervised algorithms.

The use of unsupervised methods for real-time seizure detection have also been explored recently, as in [17], where the best results are obtained for the k-means and k-mediod algorithms. Although these unsupervised algorithms do not have any dependence on training data, their classification performance is significantly lower than in the supervised case. In [18], an unsupervised method is presented that aims at easing the annotation of epileptic seizures by doctors in order to generate training data, but this method is targeted to the hospital and not to wearable devices.

In addition to the limitations in terms of data availability and classification performance, the cumbersomeness and intrusiveness of the EEG cap hinders the adoption of these methods since the majority of epilepsy patients refuse to wear this cap in their daily lives due to the negative social impact [19]. A number of studies such as [20] have been conducted in order to reduce the number of electrodes used for the detection of epileptic seizures. Some minimally invasive ultra-low energy wearable devices for recording EEG signals have been recently proposed, such as in-ear sensors [21], behind-the-ear sensors [22] or glasses with hidden electrodes [7]. These devices remove the social stigma associated with the EEG caps while retaining capability of patient monitoring.

Finally, there are works in the literature that present selfaware systems for personalized health care such as [23], [24]. Nevertheless, these systems tackle the problem of patient deterioration and not the specific problem of generating personalized training data.

To the best of our knowledge, this is the first time that a selflearning methodology for EEG seizure detection is proposed. Using a minimally supervised algorithm for labeling at the edge device, our approach combines the high classification performance of the supervised methodologies with the independence from data for pretraining of the unsupervised approaches. This is done in the context of wearable platforms with a reduced number of electrodes, such as [7], [21], [22], in order to remove any component of social stigma.

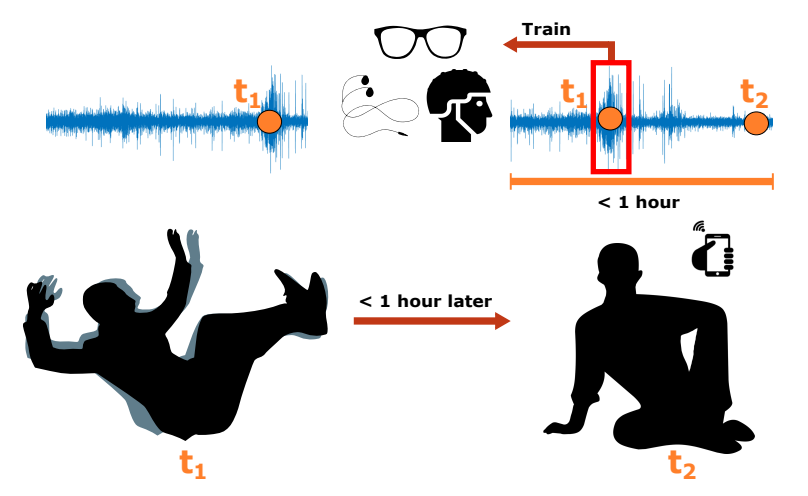

Fig. 1. Temporal pipeline for learning from undetected seizures.

\section{Methodology Overview}

In this section, we give an overview of our self-learning methodology for epileptic seizure detection. Given the negative social impact of the EEG caps mentioned above, we target non-invasive EEG wearable platforms, such as those proposed in [7], [21], [22], in which only two hidden electrodes, i.e. $F_{7} T_{3}$ and $F_{8} T_{4}$, are used to collect the EEG signals. However, our methodology is not restricted to these platforms and so, it is suitable for any wearable EEG monitoring system. The processing pipeline of our methodology is divided in three parts: feature extraction, a posteriori seizure labeling and supervised real-time seizure detection.

Fig. 1 illustrates the scenario considered in this article, i.e. our a posteriori detection algorithm is triggered by the patient after recovering from an undetected epileptic seizure and labels the position of the seizure in the signal, which is then used to train the real-time classifier.

\section{A. Feature Extraction}

EEG signals have a complex, non-stationary and nonlinear nature which hinders the use of the raw signals for epileptic seizure detection. In order to capture the most valuable information contained in the signal, we extract a number of features of power in the frequency domain and of nonlinearity. These features are extracted from four-second windows with an overlap of $75 \%$, i.e. after the features from one window are extracted, the window slides by one second. To obtain the nonlinear features, we apply the discrete wavelet transform (DWT) [12] to decompose the EEG signal until level seven using Daubechies 4 (db4) wavelet basis function.

As some of the features extracted contain redundant information, we use backward elimination [25] to sort them in order of relevance. We observed that extracting the ten most relevant features offers a proper trade-off between accuracy and complexity and therefore, the following features are extracted: total theta $([4,8] \mathrm{Hz})$ band power, relative theta band power and total delta $([0.5,4] \mathrm{Hz})$ band power from electrode $F_{7} T_{3}$ and relative theta band power, seventh level permutation entropy for $n=5$ and $n=7$ [26], sixth level permutation entropy for $n=7$, third level Renyi entropy and sixth level sample entropy for $k=0.2$ and $k=0.35$ [27] from electrode $F_{8} T_{4}$. 


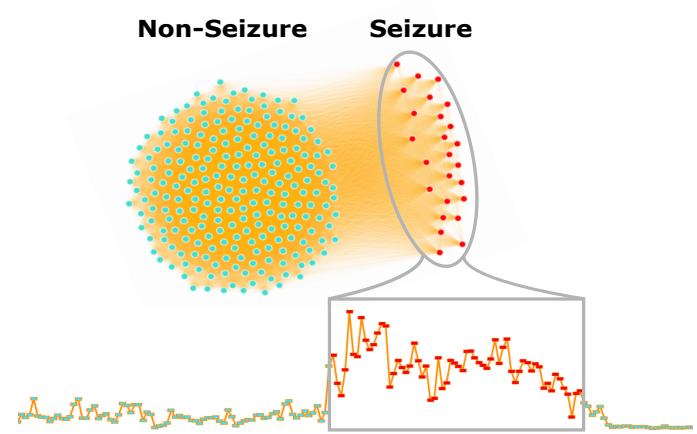

Fig. 2. Graphical representation of the algorithm. A value of distance is obtained for each sliding window in the feature space. The window with the maximum distance is labeled as seizure.

\section{B. A Posteriori Seizure Detection at the Edge Device}

To label the seizures present in the EEG signal, we propose a minimally supervised algorithm that exploits two pieces of information. The first one is the confirmation that the last hour of EEG signal contains an epileptic seizure; as mentioned above, this input is provided by the patient and is used to trigger the algorithm. The second one is the average length of the epileptic seizures, which is provided by a medical expert.

Our algorithm uses as inputs the average length of the epileptic seizures and the features, extracted as described in the previous subsection. Then, it finds the position of the epileptic seizure in the signal using a clustering scheme specifically designed for this problem. This information is used to label the epileptic seizure in the signal. An in-depth explanation of the proposed algorithm is given in Section IV.

\section{Supervised Real-Time Seizure Detection}

The labels produced at the output of the a posteriori detection algorithm are used to train a supervised real-time seizure detection algorithm that is in charge of raising the alerts in case a seizure occurs. We consider the detection algorithm proposed by the authors of [7] since its classification performance using the electrode pairs $F_{7} T_{3}$ and $F_{8} T_{4}$ has already been demonstrated. However, our methodology is not restricted to this algorithm and so, any real-time detection technique can be used.

In [7], the authors extract 54 features from the raw signal recorded at each electrode pair and use this data as the input to a classifier based on the random forest algorithm [28].

\section{Minimally SUPERVISED ALGORITHM FOR A POSTERIORI SEIZURE DETECTION}

In this section, we present our algorithm for a posteriori seizure detection at the edge device. Our algorithm exploits the inherent differences existing in the EEG signal between epileptic seizures and seizure-free parts.

Basically, the algorithm calculates a measure of the distance between a continuous set of points under a sliding window and the rest of the signal. The distance is obtained by adding the individual distances between each point inside the window and each point outside it. The algorithm is shown in pseudo-code

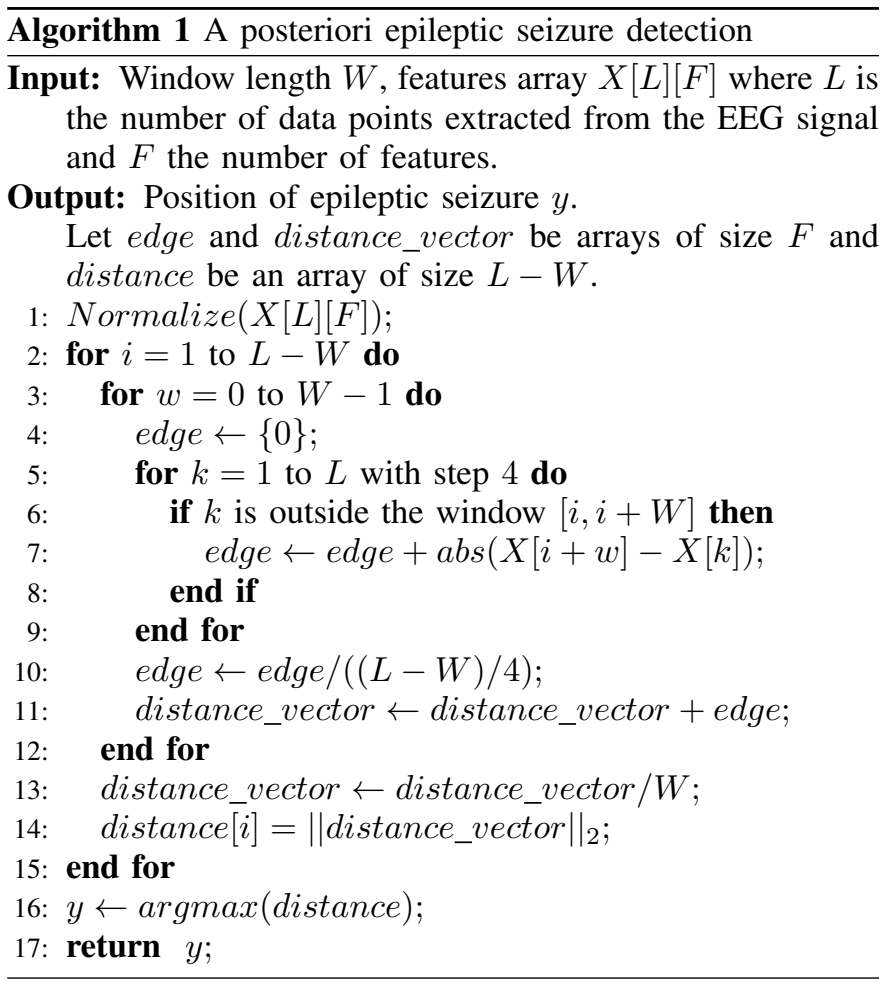

in Algorithm 1 and depicted graphically in Fig. 2. The figure shows that the window with the largest distance in the feature space is found and labeled as seizure.

The inputs of the algorithm are the window length $W$, which is the average duration of the epileptic seizures of the patient, and the features array $X$ of dimensions $L \times F$, where $L$ is the number of data points extracted from the signal and $F$ the number of features (in this case 10). In order to have all the features in the same scale, they are normalized in Line 1 of the Algorithm 1: the mean value, across the signal, of the corresponding feature is subtracted and the result is divided by the standard deviation of the feature.

Then, in the first for-loop of the algorithm, in Line 2, the window of size $W$ slides along the first dimension of the features array, i.e. the signal length, with step one. The second for-loop, in Line 3, iterates over every point inside this window. The third for-loop along with the condition in Line 6 is used to iterate over every fourth point outside of the considered window. Given the $75 \%$ overlap in the feature extraction step, by taking every fourth point, redundant information is avoided and the complexity is reduced.

At this point, the absolute differences between a point contained in the window and every fourth point of those outside the window are obtained feature by feature. In Line 7, these values are summed up and in Line 10 normalized by the total elements added, i.e. $(L-W) / 4$. The result is stored in an array called edge of size $F$. This calculation is repeated for all the $W$ points contained in the window and all of the values of the edge vector are accumulated in Line 11 in a vector of length $F$ called distance_vector. This vector is normalized over $W$ in line 13 .

In order to have a single value for the distance instead of 
$F$ values, the Euclidean norm of the vector distance_vector is calculated across features. This is done in Line 14 and it is represented by the function $\|\cdot\|_{2}$. As a result, we have a vector called distance of length $L-W$ that contains the distance value for all the $L-W$ windows of length $W$.

In Line 16, the index of the position with the maximum distance is obtained and it is set as the position of the epileptic seizure and returned as the output $y$ of the algorithm. Finally, the seizure is labeled as the points in the range $[y, y+W]$.

To calculate the complexity of the algorithm, we observe that, after extracting and normalizing the features, the algorithm considers $L-W$ windows. Each window has $W$ points with $F$ features and for each of these data points $(L-W) / 4$ subtractions are calculated. Therefore, its complexity is $O\left(L^{2} W F\right)$, which means that in a wearable platform such as the one described in Section V-B one second of signal is processed in one second time.

The algorithm we have presented in this section labels seizures at the edge device without medical supervision. This data can train the online supervised classifier enabling a selflearning pipeline for epileptic seizure detection. In Section VI we demonstrate the quality of our a posteriori seizure detection algorithm and its suitability for the proposed selflearning methodology.

\section{EXPERIMENTAL SETUP}

In this section, we present the experimental setup for evaluation of our proposed methodology. In Section V-A, the database of EEG signals considered in this article is described. In Section V-B, we present the wearable platform where we ported our algorithm for evaluation purposes. Finally, Section $\mathrm{V}-\mathrm{C}$ contains a description of the metric employed to evaluate the classification performance of the proposed algorithm.

\section{A. Epileptic Seizures Database}

To evaluate the classification performance of the proposed algorithm, we use the Physionet.org CHB-MIT Scalp EEG database. This database contains recordings of EEG signals from young patients in the range of age from 1.5 to 22 years with refractory seizures. These EEG signals are sampled at a frequency of $256 \mathrm{~Hz}$. From this database we consider traces from 9 patients that are compliant with the standard acquisition protocol [11], with a total of 45 epileptic seizures. From these traces we selected only the channels corresponding to the electrode pairs of interest in this work, i.e. $F_{7} T_{3}$ and $F_{8} T_{4}$.

\section{B. Target Wearable Platforms}

Our proposed methodology has been evaluated in a typical architecture of current EEG-based wearable platforms (e.g., glasses, in-ear or behind-the-ear platforms). In particular, the considered representative platform features an ultralow power 32-bit microcontroller STM32L151 [29] with an $\mathrm{ARM}^{\circledR}$ Cortex $^{\circledR}-\mathrm{M} 3$, whose maximum operating frequency is $32 \mathrm{MHz}$. The system acquires EEG signals from the two mentioned electrode pairs, $F_{7} T_{3}$ and $F_{8} T_{4}$, at a sampling frequency ranging from $125 \mathrm{~Hz}$ to $16 \mathrm{KHz}$ with up to 16bit resolution. The memory of this system consists of $48 \mathrm{~KB}$ RAM and $384 \mathrm{~KB}$ Flash, the battery has a capacity of 570 $\mathrm{mAh}$ and it includes a 24-bit ADC [30].

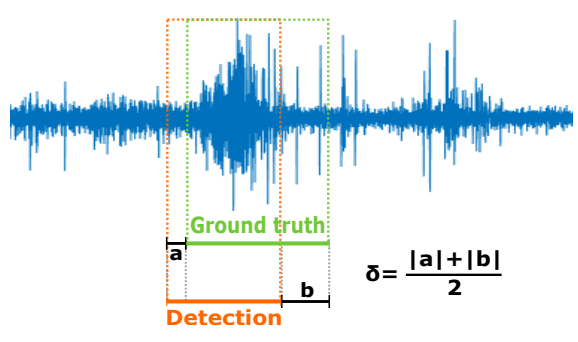

Fig. 3. Graphical representation of the proposed metric.

\section{Metric}

To have a fair measure of the classification performance of the algorithm, we propose a deviation metric $\delta$ given by the average of the absolute distances between the starting points and between the ending points of our a posteriori detection and the ground truth. Let $y$ be the ground truth and $y^{\prime}$ the position detected a posteriori, the metric is defined as:

$$
\delta=\frac{\left|y_{\text {start }}-y_{\text {start }}^{\prime}\right|+\left|y_{\text {end }}-y_{\text {end }}^{\prime}\right|}{2}
$$

This gives a non-normalized measure in seconds that provides an understanding of the combination of distance and overlap between both labels. Fig. 3 shows how the value of $\delta$ accounts for the overlap of the labels.

To compare the quality of the detection with signals of different lengths it is convenient to have a normalized measure i.e. a measure in the range $[0,1]$. In order to obtain this normalized measure, we define $N$ as the maximum error we can get from the non-normalized metric and hence, the normalized metric is defined as:

$$
\delta_{\text {norm }}=1-\frac{\left|y_{\text {start }}-y_{\text {start }}^{\prime}\right|+\left|y_{\text {end }}-y_{\text {end }}^{\prime}\right|}{2 N},
$$

where $N=\max \left(L-\frac{y_{\text {start }}+y_{\text {end }}}{2}, \frac{y_{\text {start }}+y_{\text {end }}}{2}\right)$.

\section{EXPERIMENTAL RESULTS}

This section demonstrates the classification performance of the proposed algorithm for a posteriori seizure detection and its suitability for our self-learning methodology. In Section VI-A the classification performance of our algorithm for a posteriori seizure labeling is presented, while in Section VI-B, the classification performance of a supervised classifier trained with data labeled by our algorithm is studied. Finally, in Section VI-C, the energy consumption and the battery lifetime of the target platform running our methodology are analyzed.

\section{A. Evaluation of the Classification Performance of the a Posteriori Labeling Algorithm}

To extensively evaluate the robustness of our proposed algorithm, we generate a large number of test samples. Each sample consists of an EEG signal of random duration ranging between 30 minutes and 1 hour that contains a single epileptic seizure. For each one of the 45 epileptic seizures contained in the database, 100 different samples were produced, resulting in a total of 4500 test samples. 
Our a posteriori detection algorithm using the 10 selected features was run on each of these samples and the accuracy was measured with the proposed metric. We firstly calculate the mean of the non-normalized metric and the geometric mean of the normalized metric (which is the only correct average of normalized values) [31], across the 100 samples of each seizure. Next, we extract the median values across the seizures of each patient in order to obtain a non-normalized and a normalized value per patient. Finally, we calculate the total classification performance as the median across all seizures.

The total value of classification performance computed in this manner is $\delta=10.1 \mathrm{~s}$ and $\delta_{\text {norm }}=0.9935$. The interpretation of these results is that the median detected position of the epileptic seizure is at a distance within 10.1 seconds from the true seizure or within a $1 \%$ of the total signal. To have a more accurate picture of the classification performance, Table I shows the values obtained per patient.

TABLE I. CLASSIFICATION PERFORMANCE PER PATIENT

\begin{tabular}{|c|c|c|c|c|c|c|c|c|c|}
\hline ID & $\mathbf{1}$ & $\mathbf{2}$ & $\mathbf{3}$ & $\mathbf{4}$ & $\mathbf{5}$ & $\mathbf{6}$ & $\mathbf{7}$ & $\mathbf{8}$ & $\mathbf{9}$ \\
\hline$\delta(\boldsymbol{s})$ & 14.5 & 53.2 & 5.5 & 15.9 & 5.7 & 11.5 & 13.9 & 3.2 & 5.0 \\
\hline$\delta_{\text {norm }}(\boldsymbol{\%})$ & 99.0 & 96.3 & 99.6 & 98.9 & 99.6 & 99.2 & 99.1 & 99.8 & 99.7 \\
\hline
\end{tabular}

The classification performance is higher than $95 \%$ for all patients and higher than $98.5 \%$ for all of them except for patient 2. A deeper insight into the results is given in Table II, where the mean value of $\delta$ is given for each seizure. Only three labels, one in each of patients 2, 3 and 4, are far away from the actual seizures. These three misplaced values are due to large bursts of noise in the signal near the epileptic seizure. From a global perspective, $73.3 \%$ of the seizures are detected within 15 seconds, $86.7 \%$ within 30 seconds and $93.3 \%$ within one minute from the ground truth, which indicates the high quality of our proposed algorithm.

\section{B. Validation on Supervised Real-Time Classifier}

In order to demonstrate the suitability of the algorithm for labeling epileptic seizures, we train the supervised real-time classifier based on data labeled by our a posteriori detection algorithm instead of by medical experts.

TABLE II. VALUE OF $\delta$ IN SECONDS PER SEIZURE

\begin{tabular}{|c|c|c|c|c|c|c|c|}
\hline \multirow{2}{*}{$\begin{array}{c}\text { Patient } \\
\text { ID }\end{array}$} & \multicolumn{7}{|c|}{ Seizure Number } \\
\hline & $\mathbf{1}$ & $\mathbf{2}$ & $\mathbf{3}$ & $\mathbf{4}$ & $\mathbf{5}$ & $\mathbf{6}$ & $\mathbf{7}$ \\
\hline $\mathbf{1}$ & 15 & 19 & 12 & 7 & 13 & 16 & 21 \\
\hline $\mathbf{2}$ & 19 & 373 & 53 & & & & \\
\hline $\mathbf{3}$ & 443 & 4 & 6 & 3 & 14 & 3 & 8 \\
\hline $\mathbf{4}$ & 408 & 21 & 6 & 11 & & & \\
\hline $\mathbf{5}$ & 3 & 6 & 10 & 6 & 3 & & \\
\hline $\mathbf{6}$ & 12 & 7 & 17 & & & & \\
\hline $\mathbf{7}$ & 12 & 4 & 32 & 14 & 40 & & \\
\hline $\mathbf{8}$ & 3 & 5 & 2 & 4 & & & \\
\hline $\mathbf{9}$ & 15 & 3 & 2 & 3 & 6 & 13 & 5 \\
\hline
\end{tabular}

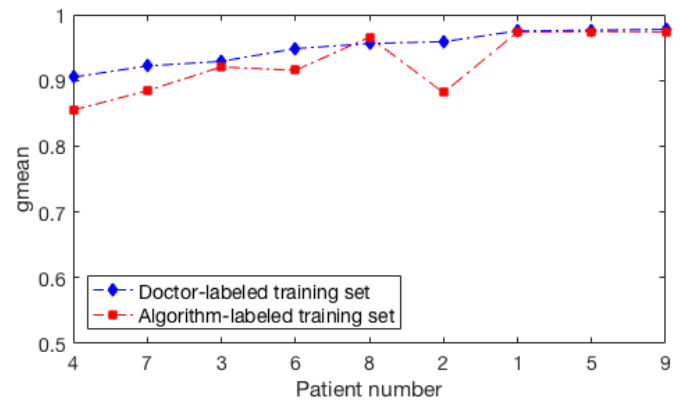

Fig. 4. Geometric mean for doctor-labeled versus algorithm-labeled training set, note that the scale starts at 0.5 for better resolution.

To evaluate our algorithm, we take for each seizure one signal with a length ranging between 30 minutes and 1 hour that contains the seizure. Then, we use our algorithm to label the seizures. This newly labeled data, together with seizurefree samples, conforms the training set of the experiments.

Then, following [7], a set of experiments with personalized data are performed where the training set is balanced and consists of 2 to 5 seizures coming from the same subject that is being tested. Thus, the length of the training set ranges between 5 and 30 minutes of EEG recordings. The results of these tests are evaluated against the data labeled by medical experts. Sensitivity, specificity and the geometric mean of the results are calculated and used for evaluation. In Fig. 4, the results of expert-labeled training data are compared with the results of algorithm-labeled training data.

These results show that when the online classifier is trained with algorithm-labeled data the classification performance is almost as high as when the labels are given by medical experts. Overall, the geometric mean across all subjects using the labels provided by medical experts is $94.95 \%$. Using our algorithm for labeling the data, the geometric mean across subjects yields $92.60 \%$, which represents a degradation of only $2.35 \%$, being the degradation in terms of sensitivity and specificity $2.43 \%$ and $2.26 \%$ respectively. Thus, the proposed algorithm is a promising labeling alternative to the costly and lengthy process of labeling data by medical experts.

\section{Energy Consumption and System Lifetime}

Since the proposed algorithm for seizure labeling is only triggered upon occurrence of an epileptic seizure, its energy consumption on the target platform depends on the frequency of the seizures. We calculate the lifetime for a frequency ranging between one seizure a month to one seizure a day, i.e. with the duty cycle of the algorithm ranging between $0.14 \%$ and $4.17 \%$. On top of this, the algorithm requires the EEG signal to be constantly sampled from the two electrode pairs and so, the EEG acquisition duty cycle is $100 \%$ and the required memory for one hour of data is $240 \mathrm{~KB}$. Under these conditions and with a battery of $570 \mathrm{mAh}$, as described in Section V-B, running only our a posteriori detection algorithm on a single battery charge allows for between 631.46 and 430.16 hours of operation, i.e. from 26.31 to 17.92 days, depending on the frequency of the epileptic seizures. 
Total Energy Consumption

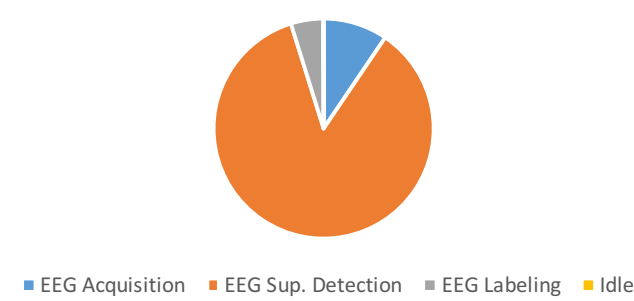

Fig. 5. Percentage of energy consumption of each task.

The supervised real-time classifier considered in this work requires three seconds for processing a four-second window and thus, the CPU duty cycle when running only the supervised detection algorithm is $75 \%$. This allows for 65.15 hours, that is, 2.71 days of operation on a single battery charge.

When both, our labeling algorithm and the online detection algorithm are run on the same platform, the lifetime of the battery ranges between 2.71 and 2.59 days, depending on the frequency of the epileptic seizures. Hence, the degradation in the battery lifetime due to the energy consumed by our a labeling algorithm is minimal. These results are summarized in Table III, and Fig. 5 shows that the online supervised detection consumes a much larger percentage of the total energy than our algorithm for EEG labeling.

TABLE III. BATTERY LIFETIME OF THE SYSTEM FOR THE WORST CASE (ONE SEIZURE PER DAY)

\begin{tabular}{|c|c|c|c|c|}
\hline Task & $\begin{array}{c}\text { Current } \\
(\boldsymbol{m A})\end{array}$ & $\begin{array}{c}\text { Duty } \\
\text { Cycle }(\boldsymbol{\%})\end{array}$ & $\begin{array}{c}\text { Avg. current } \\
(\boldsymbol{m A})\end{array}$ & $\begin{array}{c}\text { Energy } \\
(\boldsymbol{\%})\end{array}$ \\
\hline EEG Acquisition (x2) [30] & 0.870 & $100 \%$ & 0.870 & 9.47 \\
EEG Sup. Detection & 10.5 & $75 \%$ & 7.875 & 85.72 \\
EEG Labeling & 10.5 & $4.17 \%$ & 0.438 & 4.77 \\
Idle & 0.018 & $20.83 \%$ & 0.004 & 0.04 \\
\hline Battery Lifetime & $\mathbf{2 . 5 9}$ days & \multicolumn{3}{|l}{} \\
\cline { 1 - 3 }
\end{tabular}

\section{CONCLUSION}

In this work, we have conceived a self-learning methodology for epileptic seizure detection and proposed a novel algorithm for labeling epileptic seizures at the edge device without medical supervision. In this manner, our labeling algorithm generates personalized data that is used to train the real-time classifier. Our experiment shows that the proposed algorithm can run on non-invasive EEG-based wearable platforms together with an online classifier during between 2.71 and 2.59 days on a single battery charge. We demonstrated that the median deviation of the labels from the ground truth is only 10.1 seconds or, equivalently, less than $1 \%$ of the signal length. Moreover, when our algorithm is used for labeling instead of a medical expert, the classification performance of a real-time detection algorithm degrades by only $2.35 \%$. Thus, the proposed algorithm is effective in removing the need for medical experts to record and label epilepsy data, which eases the adoption of wearable seizure monitoring systems and helps to improve the quality of life of epilepsy patients.

\section{REFERENCES}

[1] "About Epilepsy: The Basics - Epilepsy Foundation," 2014. [Online]. Available: https://www.epilepsy.com/learn/about-epilepsy-basics

[2] D. Hirtz et al., "How common are the "common" neurologic disorders?" Neurology, vol. 68, 2007.

[3] H. Blumenfeld, "Impaired consciousness in epilepsy." The Lancet. Neurology, vol. 11, no. 9, pp. 814-26, sep 2012.

[4] A. Szucs et al., "Sudden death and mortality in epilepsy." Ideggyogyaszati szemle, 2006.

[5] G. D. Cascino, "Video-EEG Monitoring in Adults," Epilepsia, 2002.

[6] T. N. Alotaiby, S. A. Alshebeili, T. Alshawi, I. Ahmad, and F. E. Abd El-Samie, "Eeg seizure detection and prediction algorithms: a survey," EURASIP Journal on Advances in Signal Processing, 2014.

[7] D. Sopic, A. Aminifar, and D. Atienza, "e-glass: A wearable system for real-time detection of epileptic seizures," in 2018 IEEE International Symposium on Circuits and Systems (ISCAS), May 2018.

[8] "After a Seizure - Epilepsy Scotland." [Online]. Available: http://www.epilepsyscotland.org.uk/pdf/After_a_seizure.pdf

[9] J. E. Allen, C. D. Ferrie, J. H. Livingston, and R. G. Feltbower, "Recovery of consciousness after epileptic seizures in children," Archives of Disease in Childhood, 2007.

[10] J. Blom and M. Anneveldt, "An electrode cap tested," Electroencephalography and Clinical Neurophysiology, 1982.

[11] "10-20 system (EEG) - an overview - ScienceDirect Topics."

[12] H. Ocak, "Automatic detection of epileptic seizures in EEG using discrete wavelet transform and approximate entropy," Expert Systems with Applications.

[13] G. Chen, "Automatic eeg seizure detection using dual-tree complex wavelet-fourier features," Expert Systems with Applications, 2014.

[14] J. Yoo et al., "An 8-channel scalable eeg acquisition soc with patientspecific seizure classification and recording processor," IEEE Journal of Solid-State Circuits, 2013.

[15] Y. Kumar, M. L. Dewal, and R. S. Anand, "Epileptic seizures detection in eeg using dwt-based apen and artificial neural network," Signal, Image and Video Processing, 2014.

[16] U. R. Acharya, S. L. Oh, Y. Hagiwara, J. H. Tan, and H. Adeli, "Deep convolutional neural network for the automated detection and diagnosis of seizure using eeg signals," Computers in Biology and Medicine, 2018.

[17] O. Smart and M. Chen, "Semi-automated patient-specific scalp eeg seizure detection with unsupervised machine learning," in 2015 IEEE Conference on Computational Intelligence in Bioinformatics and Computational Biology (CIBCB), Aug 2015.

[18] K. M. Tsiouris, S. Markoula, S. Konitsiotis, D. D. Koutsouris, and D. I. Fotiadis, "A robust unsupervised epileptic seizure detection methodology to accelerate large eeg database evaluation," Biomedical Signal Processing and Control, 2018.

[19] C. Hoppe et al., "Novel techniques for automated seizure registration: Patients' wants and needs," Epilepsy \& Behavior, 2015.

[20] F. Fürbass et al., "Automatic multimodal detection for long-term seizure documentation in epilepsy," Clinical Neurophysiology, 2017.

[21] V. Goverdovsky et al., "Hearables: Multimodal physiological in-ear sensing," Scientific Reports, 2017.

[22] Y. Gu et al., "Comparison between scalp eeg and behind-the-ear eeg for development of a wearable seizure detection system for patients with focal epilepsy," Sensors (Basel), 2018.

[23] A. Anzanpour et al., "Self-awareness in remote health monitoring systems using wearable electronics," in Proceedings of the Conference on Design, Automation \& Test in Europe, 2017.

[24] F. Forooghifar, A. Aminifar, and D. Atienza Alonso, "Self-aware wearable systems in epileptic seizure detection," in Euromicro Conference on Digital System Design (DSD), no. CONF, 2018.

[25] P. A. Devijver and J. Kittler, Pattern recognition: A statistical approach. Prentice Hall, 1982.

[26] C. Bandt and B. Pompe, "Permutation Entropy: A Natural Complexity Measure for Time Series," Physical Review Letters, 2002.

[27] X. Xinnian Chen, I. Solomon, and K. Chon, "Comparison of the Use of Approximate Entropy and Sample Entropy: Applications to Neural Respiratory Signal," in 2005 IEEE Engineering in Medicine and Biology 27th Annual Conference. IEEE, 2005.

[28] R. Diaz-Uriarte and S. A. de Andres, "Gene selection and classification of microarray data using random forest," BMC Bioinformatics, 2006.

[29] "STM32L1 Series - STMicroelectronics."

[30] "ADS1299-4 Low-Noise, 4-Channel, 24-Bit Analog-to-Digital Converter for Biopotential Measurements - TI.coms."

[31] P. J. Fleming and J. J. Wallace, "How not to lie with statistics: The correct way to summarize benchmark results," Commun. ACM, 1986 